\title{
Confirmatory Factor Analysis of the Youth Experiences Survey for Sport (YES-S)
}

\author{
Philip J. Sullivan*, Kaitlyn LaForge-MacKenzie, Matthew Marini \\ Department of Kinesiology, Brock University, St. Catharines, Canada \\ Email: "phil.sullivan@brocku.ca
}

Received 12 January 2015; accepted 4 August 2015; published 7 August 2015

Copyright (C) 2015 by authors and Scientific Research Publishing Inc.

This work is licensed under the Creative Commons Attribution International License (CC BY). http://creativecommons.org/licenses/by/4.0/

(c) (i) Open Access

\begin{abstract}
Research on Positive Youth Development (PYD) has been hampered by lack of a valid measure for the construct, particularly for research in sport. The Youth Experiences Scale for Sport (YES-S) [1], is a five-factor measure of positive youth outcomes specifically designed for the sport context. The YES-S is a promising instrument that fills an important niche in PYD research, and MacDonald et al. provided support for many of its psychometric properties. However, the factor structure of the scale is currently based on an exploratory factor analysis and has not yet been subjected to a confirmatory factor analysis (CFA). The present study was designed to confirm the five-factor structure of the YES-S. A sample of 350 youth sport athletes (196 male, 153 female) completed the YES-S. A CFA showed that a modified version of the five-factor YES-S had excellent fit of the model to the data. An analysis of invariance showed no differences in responses in terms of gender. It is concluded that this short-form YES-S offers excellent psychometric properties while retaining the original factor structure of the YES-S. The results offer further support for the validity of the factor structure of the YES-S while providing a shorter version of the scale, which may be appealing for research with younger sport participants.
\end{abstract}

\section{Keywords}

Positive Youth Development, Sport, Confirmatory Factor Analysis

\section{Introduction}

The positive outcomes that youth can develop through sport may outweigh the sport-specific outcomes athletes derive. However, the significant applied findings that research may generate are hampered by the lack of a valid measurement. Although issues with measurement validity are a characteristic of Positive Youth Development

\footnotetext{
${ }^{*}$ Corresponding author.
}

How to cite this paper: Sullivan, P.J., LaForge-MacKenzie, K. and Marini, M. (2015) Confirmatory Factor Analysis of the Youth Experiences Survey for Sport (YES-S). Open Journal of Statistics, 5, 421-429. 
(PYD) research in general, it is particularly relevant to the context of sports.

In the past, research on the development of youth and adolescents was considered to be more focused on negativity and negative outcomes. Larson (2000) [2] further investigated this idea with research looking at why youth seemed to be "trapped in the present" (p. 170), suffered from high rates of boredom and disconnected from meaningful challenges. His findings led towards the idea that these deficits were not due to psychopathology or increases in negative outcomes, but instead due to a lack of positive outcomes and positive development [2]. Therefore a new theoretical framework termed PYD was constructed to target development as an asset towards fostering positive outcomes and also reducing negative outcomes [1] [2].

The PYD framework allows for a more open-minded approach to researching and observing PYD and how the environments that we put our youth in, shape their development [1] [3] [4]. PYD is perpetuated by providing structured activity programs, such as performance and fine arts clubs, sports, academic clubs and organizations, and community organizations [5]. These programs promote well-being, help reduce boredom by allowing youth to focus on attending their weekly scheduled programs as well as reduce and prevent negative development. Youth who experience PYD can be considered as exemplary young people who are healthy and thriving [5]. Their actions benefit the well-being of themselves, parents, peers, the community, and society.

The majority of PYD studies to date have used Larson's conceptualization of the construct. Dworkin, Larson, and Hansen (2003) [6] examined growth experiences (i.e., "experiences that teach you something or expand you in some way, that give you new skills, new attitudes, or new ways of interacting with others” p. 20) through participation in organized activities (including sport). Via focus groups with 55 adolescents, they identified six domains of learning experiences that were divided into personal and interpersonal development. Personal development described processes that occurred within the individual and included three domains: identity work, development of initiative, and emotional regulation. Interpersonal development described processes that involved developing social connections and included the domains of teamwork and social skills, positive relationships including acquiring prosocial norms and diverse peer relationships, and development of adult networks and social capital.

The Youth Experiences Survey (YES) is an operationalization of Larson and colleagues’ perspective of PYD and the measure has been subjected to an on-going validation process. The YES was originally based on the qualitative findings of the Dworkin et al.'s (2003) study and vetted through additional focus groups with adolescents, refined, and subjected to review with a panel of 10 adult experts. The original measure, the YES 1.0, included 90 items which measured six major domains of positive development (i.e., identity work, initiative, basic skills, teamwork and social skills, interpersonal relationships, and adult networks) and one major domain of negative experiences. This measure was designed to be applicable to all organized activities for youth and adolescents.

As a specific organized youth activity, sport is a popular context for PYD research. Organized sport in particular has been found as an ideal environment to foster PYD [7]. In particular, youth who are involved in a structured sport environment are found to be more engaged and more intrinsically motivated as compared to youth who participate in unstructured programs in sport, leisure activities, or school [8]. Using the YES 1.0, Hansen, Larson, and Dworkin (2003) [9] found that sport activities were positively associated with moderately higher rates of personal development (i.e., self-knowledge, goal setting, effort, time management, emotional regulation, and physical skills) and interpersonal development (i.e., teamwork, social skills, leadership, and peer relations) experiences in comparison to other youth activities. Self-referenced competencies and task-oriented sporting climates have been found to be positively associated with positive factors of a sport-specific YES measure whereas other-referenced competencies and ego-oriented sporting climates were associated with negative PYD outcomes [1].

Following their work using sport-specific environments, Hansen and Larsen further refined the YES into the Youth Experiences Survey 2.0 (YES 2.0) which was revised to be shorter than the original YES but had much stronger evidence of reliability and validity [5]. Larson, et al., [10] tested the YES 2.0 across three different program-based activities in 2280 youth participants: faith-based activities, performance and fine arts activities, and sports. They found that the sports and performance and fine arts programs provided the most beneficial PYD outcomes, although sports also provided more high stress situations.

Combined, these findings suggest there are distinct learning experiences related to different youth activities. However, there have been consistent concerns about the psychometric properties of the YES 2.0, particularly when it is used in sporting contexts [1] [11]. These concerns date back to Hansen and Larson's [5] introduction 
of the YES 2.0. In their unpublished paper, the authors described the confirmatory factor analyses (CFA) procedures they used to support their scale, but omitted or neglected many of the conventional criteria for an effective procedure. In their paper, Hansen and Larson reported minimal information on the procedures used and fit of the model to the data. They compared two models for positive and negative factors independently despite a conceptual background that considered them interrelated. Specifically they compared a one-factor model of positive PYD with a model comprised of six related positive factors, and five-factor and one-factor models for negative factors. They only reported the Goodness of Fit Index (GFI), and did not mention any other global goodness of fit indices or residual-based goodness of fit indices. Furthermore, they reported no analyses for a model that included all scales. Their results for their best fit for the positive scales, which was comprised of six separate factors, was a GFI of 0.73 , which is far below the accepted value for an excellent fit of the model to the data. The one-factor model for the negative factor did show a GFI of 0.92 .

Research using the YES 2.0, particularly in sport, has regularly reported issues with the psychometric properties of the scale including the nature of the factor structure and internal reliability. Reported estimates of Cronbach's alphas for scales of the YES 2.0, which should be above 0.70 to indicate acceptable internal consistency, have ranged as low as 0.55 [11] [12].

MacDonald et al., (2012) further examined the validity and reliability of the YES 2.0 for use in sport contexts. A sample of 637 athletes aged from 9 to 19 years old participated in the study. The authors first attempted to replicate Hansen and Larson's (2005) results by testing the same four models (i.e., one positive factor, six positive factors, one negative factor, and five negative factors). They also attempted to extend on the CFA work of Hansen and Larson by testing three more complete models: a two factor model comprised of one positive and one negative factor, a seven factor model comprised of six positive and one negative factor, and an eleven factor model comprised of six positive and five negative factors. When the results did not support Hansen and Larson's model, the authors proceeded to perform exploratory factor analyses. These analyses revealed a five-factor model, which they termed the Youth Experiences Survey for Sport (YES-S). This model, which included only 37 of the original YES items, was comprised of five factors. Personal and Social Skills refers to positive relations with peers, teammates and adults. Cognitive Skills refers to how athletes develop creative and problem solving skills whereas Goal Setting measures skills involved in learning to set and achieve challenging standards. Initiative refers to high levels of attention and effort indicative of intrinsic motivation. Finally, the sole negative factor of Negative Experiences comprises a variety of anti-social and stressful experiences, choices, and influences.

The YES-S showed good fit to MacDonald et al.'s (2012) data, and appeared to be promising as a model of positive youth development specific to sporting contexts [13]. However, there are no published studies to date that confirm this factor structure. More research is needed to support the psychometric properties of the scale, particularly in the context of the previous ambivalent support that has been seen for the YES 2.0.

Given the ambivalent history of psychometric properties for the YES, specifically the confusion surrounding its factor structure and the suggestion for a sport-specific version of PYD outcomes [1] [13], the primary objective of the current study was to confirm the five-factor structure of the YES-S.

\section{Method}

\subsection{Participants}

A sample of 350 team sport athletes (196 male, 153 female, and 1 participant did not indicate gender) participated in this study. The athletes ranged in age from $13-18$ years $(M=15.41, S D=1.30)$. Their sport experience ranged from 1 - 15 years $(M=7.96, S D=2.81)$. The sports played were basketball $(n=124)$, hockey $(n=114)$, and soccer $(n=112)$. All players from each sport played in the same league. All leagues were recreation, or "house leagues", and each of these leagues served a medium-sized urban area.

\subsection{Procedures}

Upon receiving institutional review board clearance, researchers contacted youth sport league administrators via their publically available contact information. The league administrators were asked to forward a message to the coaches containing an explanation of the study and the investigators' contact information. Once the interested coaches replied to the message, an investigator attended a team meeting or practice to explain the nature of the 
study to their athletes. Interested athletes were given a questionnaire package that included a letter of invitation and a consent form for their parents to sign. Athletes were instructed to bring the package home, and, if their parents consented, to complete the survey. The surveys were collected at the following practice by the investigator. Every athlete who took a package completed and returned the survey. Completion of the survey took between 20 - 30 minutes. No athletes reported any difficulty completing the survey. All athletes completed the survey in the second half of their season to ensure they had sufficient opportunities to develop perceptions of their coaches' behaviors.

\subsection{Instruments}

The YES-S is a 37-item scale that assesses personal and interpersonal developmental experiences and negative experiences in structured activities on five major scales (4 positive and 1 negative), all measured on a 4-point scale from 1 (yes, definitely) to 4 (not at all) [1]. The 37 items of the YES-S are a subset of the 70 items of the YES 2.0, with some modifications on the phrasing of the items. Because data collection for the present study was conducted prior to the publication of MacDonald et al.'s paper, the YES 2.0 was used for data collection but the 37 items that comprised the YES-S were used for data analysis. There were some minor word choice differences on six items. For example, the YES-S item "learned a way to reach my goals" was phrased "learned to find ways to achieve my goals”. For use in the current study, at the top of the YES-S, respondents were instructed to answer the questions with specific reference to their primary sport involvement. The positive scales comprised Personal and Social Skills, which was measured by 14 items (e.g., became better at taking feedback), Initiative Experiences (4 items, e.g., learned to push myself), Cognitive Skills (5 items, e.g., improved creative skills), and Goal Setting (4 items, e.g., learned to find ways to reach my goals). The sole negative factor was termed Negative Experiences, which was measured by 10 items (e.g., felt left out).

\section{Results}

Prior to any analyses, the data set was examined for the assumptions of multivariate data analysis [14]. There was some missing data, but this was less than $1 \%$ of the complete data set, so these cells were replaced with variable means [15]. The variables of the YES-S were normally distributed (see Table 1), with the exception of the items regarding Negative Experiences. These values were retained in the data because CFA using Maximum Likelihood method is considered robust for these violations and skewed values on these items, which indicate extreme negative outcomes, are meaningful; high negative skew in these items would indicate that they rarely happen. There were no instances of multicollinearity $(r>0.90)$ in the data set. Finally, because Mardia's normalized coefficient for multivariate kurtosis (5.38) was significant, analyses were based on the Satorra-Bentler scale chi-square statistic [16], which corrects for $\chi^{2}$ when distributional assumptions are not met.

The current data was subjected to a CFA with the five-factor model of the YES-S. This initial model produced indices of fit including S-B $\chi_{(619)}^{2}=1480.97, \mathrm{CFI}=0.79, \mathrm{SRMR}=0.077$, and $\mathrm{RMSEA}=0.063$. Although values of .95 and greater on the CFI have been proposed as indicative of excellent fit of the model to the data [17], it has been argued that this is too restrictive and values of $0.92-0.94$ may be considered as evidence of excellent fit [18]. Accepted criteria for excellent fit are 0.08 or less for the SRMR [17], and 0.10 or less for the RMSEA [19]. As these fits did not approach an excellent fit of the model to the data, it was decided to modify the model in an attempt to improve the fit. Specific suggestions have been made for model modifications [20]. First, they suggest that factor loadings less than 0.50 be eliminated from the model, which suggested the reduction of the model by five items. This second model showed much better fit indices $\left(\mathrm{S}-\mathrm{B} \quad \chi_{(452)}^{2}=975.74\right.$, CFI $=$ 0.84 , SRMR $=0.059$, RMSEA $=0.058$ ). Traditionally, examination of a significant difference between models involves comparing the difference in $\chi^{2}$. However, use of the S-B $\chi^{2}$ is slightly more complicated as this difference is not distributed as a $\chi^{2}[21]$. Furthermore, the $\Delta \chi^{2}$ test has recently been criticized as both impractical and unrealistic [22] [23]. The use of a $\Delta$ CFI test for model equivalence has been suggested, since changes in CFI $>$ 0.01 support the conclusion that models differ significantly [22]. According to this criteria, this second model was a significantly better fit to the data $(\triangle C F I=0.05)$ than the original YES-S model. However, this model did not yet show results of a consistent excellent fit of the model to the data, so further modifications were attempted. Although Hair et al. suggested that factor loadings should be at least 0.50 , factor loadings of at least 0.70 are ideal. This resulted in a third model with the elimination of 13 more items. However, three of these would have resulted in the factors of Goal Setting, Initiative and Cognitive Skills being reduced to three items, 
Table 1. Descriptive statistics and standardized factor loadings of items.

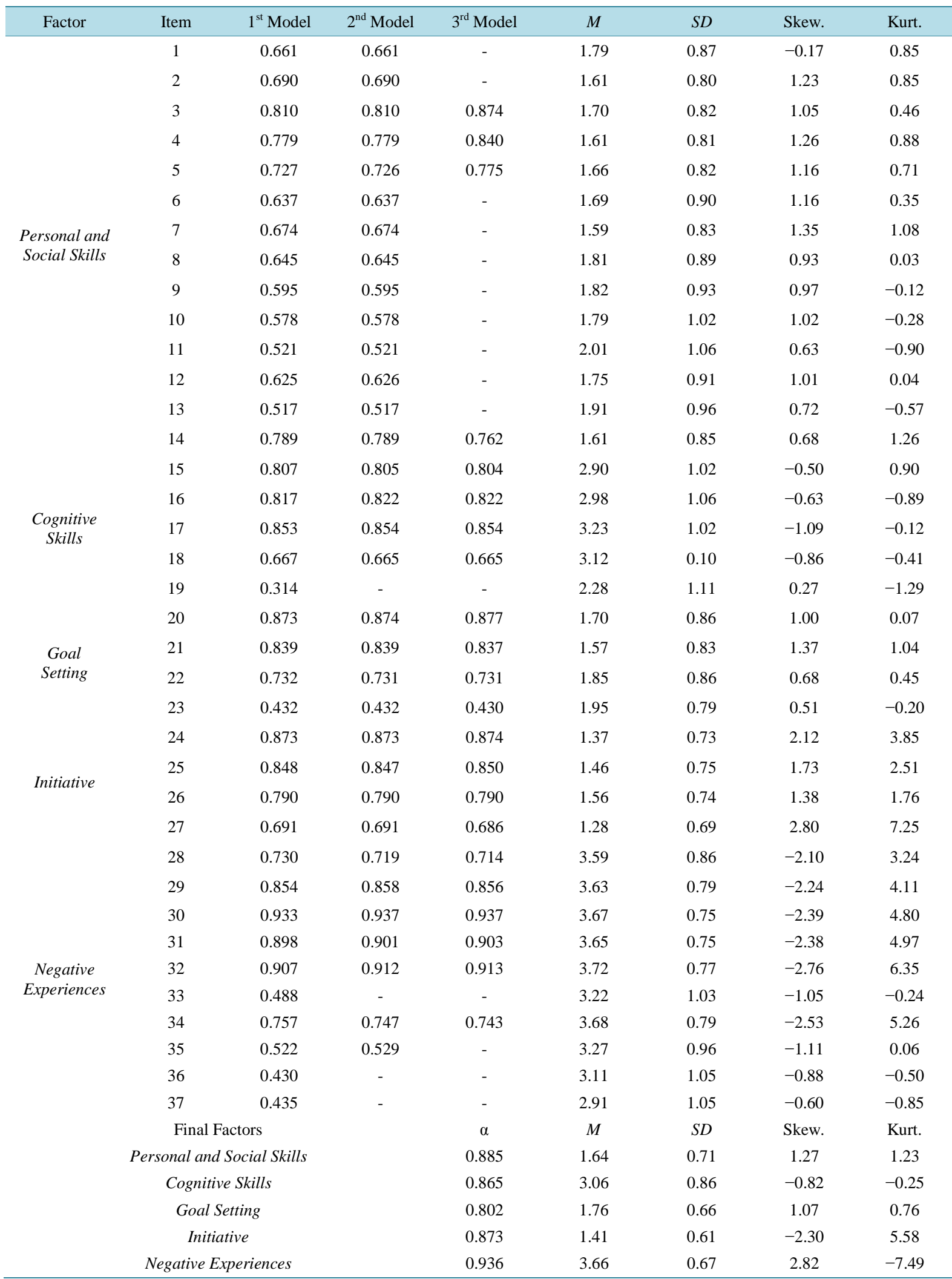

Note: The latent factors are italicized. All loadings were standardized and statistically significant $(p<0.05)$. Skew $=$ skewness. Kurt $=$ kurtosis. 
and Hair et al. suggest that each factor contain at least four items. Therefore these three variables were retained, and the third model had ten less variables than the second model. This third model showed excellent goodness of

fit statistics $\left(\mathrm{S}-\mathrm{B} \chi_{(197)}^{2}=323.37\right.$, CFI $=0.94$, SRMR $=0.054$, RMSEA $=0.043$ ) and was a significant improvement on the second model $(\triangle \mathrm{CFI}=0.10)$. These models are summarized in Table 1.

In addition to the excellent fit of the model to the data, the factors of the third model also showed excellent reliability; each factor fulfilled the reliability criteria of Hair et al. (2006) of comprising at least four items and Cronbach's alphas ranged from 0.802 - 0.936 (see Table 1). The final model included 22 items. Personal and Social Skills, Cognitive Skills, Goal Setting and Initiative were all represented by 4 items, Negative Experiences was represented by 6 items. The items and factors for the revised YES-S are given in Appendix A.

Because the current sample consisted of relatively equal numbers of males and females, an analysis of invariances was conducted to see if the five-factor model was a good fit for both genders. Analysis of invariance is a set of hierarchically ordered and increasingly restrictive tests of equality between groups. This analysis comprises (1) establishing the model for both genders individually, (2) testing the model with both groups simultaneously (in what is called a configural mode), (3) examining the invariance of factor loadings between genders (in a measurement equivalent model), and finally (4) examining the invariance of factor variances and covariances between genders (in a structural equivalence model).

The measurement model revealed good fit as a baseline model for both male $\left(\mathrm{S}-\mathrm{B} \chi_{(199)}^{2}=351.09\right.$, CFI $=$ $0.90, \mathrm{SRMR}=0.061, \mathrm{RMSEA}=0.063)$ and female $\left(\mathrm{S}-\mathrm{B} \quad \chi_{(199)}^{2}=249.80\right.$, CFI $=0.91$, SRMR $=0.073$, RMSEA $=0.041$ ) samples. All factor loadings were significant in both models. A test for configural equivalence through a simultaneous multi-group model also showed good fit of the model to the data $\left(\mathrm{S}-\mathrm{B} \chi_{(398)}^{2}=592.68\right.$, CFI $=$ 0.90$, SRMR $=0.067$, RMSEA $=0.053)$. At this point, it appears that the factorial structure of the YES-S is well represented by the 22-item, five-factor model with the factor loadings specified in the CFA.

The third step in the analysis of invariance, which restrained variance of factor loadings between the two samples showed the following goodness of fit: $\mathrm{S}-\mathrm{B} \chi_{(145)}^{2}=611.89, \mathrm{CFI}=0.90, \mathrm{SRMR}=0.070, \mathrm{RMSEA}=$ 0.053. Finally, the model that constrained both factor loadings and covariances showed the following results: $\mathrm{S}-\mathrm{B} \chi_{(425)}^{2}=649.60, \mathrm{CFI}=0.89, \mathrm{SRMR}=0.167, \mathrm{RMSEA}=0.055$.

Testing for invariance incorporates nested models. Therefore any given model cannot exceed the fit of the immediate previous model. According to the $\triangle$ CFI test, the factor loadings and covariances of the present model do not significantly change between groups, and it can be concluded that the 22-item, five-factor model of the YES-S is invariant across genders for adolescent athletes.

\section{Discussion}

The current study was conducted to confirm the factor structure of the YES-S as established by MacDonald et al. (2012) [1]. A CFA confirmed the five-factor model of the YES-S, although it supported a reduction in the number of items within that structure. Furthermore, this resulted in a revised form of the YES-S, which showed excellent internal consistency and did not vary according to gender in the current sample.

The current results supported the previous [1] conclusion that the five factors of Personal and Social Skills, Cognitive Skills, Initiative, and Negative Experiences comprised the most valid operational definition of PYD in the sports context. Compared to previous factor structures (e.g., combinations of six positive factors and one or five negative factors), this five-factor model has now been shown to have strong fit to the data in both the present and MacDonald et al.'s samples. Additional, the factors have been shown to be internally consistent in both samples.

The current results also extended previous work [1] in several ways. Whereas the model generated by MacDonald and colleagues was only the result of exploratory data analysis, it has now been confirmed through a CFA with a separate, although similar, sample. Furthermore, the present results have shown that the five-factor model is equivalent for both male and female athletes. This extends the value of the scale in applied research settings. In addition, it is noteworthy to reiterate that the history of research in PYD has been marred by measures with questionable psychometric properties. By providing this psychometric support for the YES-S, we have offered substantial further support for the scale as an operational definition for the construct of PYD within sport.

One interesting implication is that the current results suggest that a minimal number of items are needed to effectively measure PYD in sport. Whereas the original exploratory analysis supported a total of 37 items [1], the final CFA results were based on 22 items. Ten of the items that were deleted were from the factor of Personal 
and Social Skills, and four of the remaining five deletions were from Negative Experiences. Originally, these two factors contained 23 of the total 37 items on the YES-S. The results of the analysis may suggest that many items were not needed for these factors to comprise a psychometrically sound measure. It should also be noted that four other items in the remaining three factors of Cognitive Skills, Goal Setting, and Initiative could have been eliminated using the same criteria that resulted in the above decisions if not for the further restriction of needing at least four items per factor. However, the final scale, with a more even number of items per factor than the original YES-S definitely showed the best fit of the model to the data in the current sample.

Based on the current results, we would suggest that the 22-item version of the YES-S is a valid and psychometrically sound measure of PYD in sport. This version of the scale may be more appealing to researchers than the YES-S because it is more concise than the original YES-S, which can be a benefit in research with youth. Furthermore, our current results also support the five-factor scale underlying the YES-S [1], suggesting consistency in the five-factor structure across youth samples and sports.

Considering the history of the YES as a scale which has already undergone several revisions (i.e., the YES, YES 2.0, YES-S) and the need for minimizing confusion in the literature, we suggest the current scale be referred to as the "short-form YES-S". The current study should also be understood within the context of the development of a sport-specific instrument to measure PYD. The YES 2.0 was in itself a response to the need of a more psychometrically sound measure than the YES, specifically for research within sport [9] [11]. In addition to the significance of the current study in the continued development of a sport-specific measure of PYD, it is important to recognize both the statistical and applied implications of the short-form YES-S. The original form of the YES was a total of 90 items, measuring a variety of domains of PYD in various organized activities [9]. In moving to the YES 2.0, Hansen and Larsen (2005) shortened the scale to 70 items, however still focusing on a number of organized youth activities. This was subsequently reduced to 37 [1], focusing only on experiences in sports. Finally, the current study again reduced the items, keeping the focus solely on sport activities. In eliminating these items, the resulting short-form YES-S shows excellent psychometric characteristics, resulting in a concise, psychometrically sound instrument. So while the short-form YES-S fits the same factor structure found by MacDonald et al., it does so with a smaller number of items which may be an appealing applied characteristic when researching youth sport.

The limitations of the current study include that a large number of changes were made to the short-form YES-S based on one sample. Although these changes resulted in the retention of the five-factor model of the original YES-S, a more balanced version of the scale, and the demonstration of excellent psychometric properties, we recognize that these changes were based solely on one study. In addition, only a small number of sports were examined: basketball, hockey, and soccer. These sports were all interactive team sports. As a result of these limitations, we suggest that future studies continue to validate the short-form YES-S with a variety of samples, including sports, ages, and contexts (e.g., competitive vs. non-competitive and team vs. individual sports).

\section{Conclusion}

In conclusion, the short-form YES-S offers excellent psychometric properties while retaining the conceptualization of PYD [1]. As such, even with the deletion of items with lower factor loadings, the five-factor structure of PYD in sport and the theme of each of these factors remain. The short-form YES-S offers a viable and psychometrically sound alternative to the YES-S as, from an applied perspective, the short-form YES-S may be used in conjunction with other measures to fully capture youth experiences in sport. We suggest that future studies further examine the psychometric properties of short-form YES-S with a variety of sports, ages, and contexts.

\section{References}

[1] MacDonald, D.J., Côté, J., Eys, M. and Deakin, J. (2012) Psychometric Properties of the Youth Experience Survey with Young Athletes. Psychology of Sport and Exercise, 13, 332-340. http://dx.doi.org/10.1016/j.psychsport.2011.09.001

[2] Larson, R.W. (2000) Toward a Psychology of Positive Youth Development. American Psychologist, 55, 170-183. http://dx.doi.org/10.1037/0003-066X.55.1.170

[3] Lerner, R.M., Almerigi, J.B., Theokas, C. and Lerner, J.V. (2005) Positive Youth Development. Journal of Early Adolescence, 25, 10-16. http://dx.doi.org/10.1177/0272431604273211 
[4] Lerner, R.M. (2005) Promoting Positive Youth Development: Theoretical and Empirical Bases. Workshop on the Science of Adolescent Health and Development, National Research Council/Institute of Medicine, Washington DC, 9 September 2005, 92 p.

[5] Hansen, D.M. and Larson, R. (2005) The Youth Experience Survey 2.0: Instrument Revisions and Validity Testing. Unpublished Manuscript, University of Illinois at Urbana-Champaign.

[6] Dworkin, J.B., Larson, R. and Hansen, D. (2003) Adolescents' Accounts of Growth Experiences in Youth Activities. Journal of Youth and Adolescence, 32, 17-26. http://dx.doi.org/10.1023/A:1021076222321

[7] Fraser-Thomas, J.L., Côté, J. and Deakin, J. (2005) Youth Sport Programs: An Avenue to Foster Positive Youth Development. Physical Education \& Sport Pedagogy, 10, 19-40. http://dx.doi.org/10.1080/1740898042000334890

[8] Wilkes, S. and Cote, J. (2010) The Developmental Experiences of Adolescent Females in Structured Basketball Programs. Revue phénEPS/PHENex Journal, 2, 1-21.

[9] Hansen, D.M., Larson, R.W. and Dworkin, J.B. (2003) What Adolescents Learn in Organized Youth Activities: A Survey of Self-Reported Developmental Experiences. Journal of Research on Adolescence, 13, 25-55. http://dx.doi.org/10.1111/1532-7795.1301006

[10] Larson, R.W., Hansen, D.M. and Moneta, G. (2006) Differing Profiles of Developmental Experiences across Types of Organized Youth Activities. Developmental Psychology, 42, 849-863. http://dx.doi.org/10.1037/0012-1649.42.5.849

[11] Gould, D. and Carson, S. (2011) Young Athletes’ Perceptions of the Relationship between Coaching Behaviors and Developmental Experiences. International Journal of Coaching Science, 5, 3-29.

[12] Strachan, L., Cote, J. and Deakin, J. (2009) An Evaluation of Personal and Contextual Factors in Competitive Youth Sport. Journal of Applied Sport Psychology, 21, 340-355. http://dx.doi.org/10.1080/10413200903018667

[13] Gould, D. and Carson, S. (2008) Life Skills Development through Sport: Current Status and Future Directions. International Review of Sport and Exercise Psychology, 1, 58-78. http://dx.doi.org/10.1080/17509840701834573

[14] Tabachnick, B.G. and Fidell, L.S. (2007) Using Multivariate Statistics. 5th Edition, Pearson Allyn and Bacon, Boston.

[15] Hair, J., Black, W., Babin, B. and Anderson, R. (2010) Multivariate Data Analysis. 7th Edition, Pearson Prentice Hall, Upper Saddle River.

[16] Satorra, A. and Bentler, P.M. (1994) Corrections to Test Statistics and Standard Errors in Covariance Structure Analysis. In: von Eye, A. and Clogg, C.C., Eds., Latent Variables Analysis: Applications for Developmental Research, Sage, Thousand Oaks, 399-419.

[17] Hu, L. and Bentler, P.M. (1999) Cutoff Criteria for Fit Indexes in Covariance Structure Analysis: Conventional Criteria versus New Alternatives. Structural Equation Modeling, 6, 1-55. http://dx.doi.org/10.1080/10705519909540118

[18] Byrne, B.M. (2008) Testing for Multigroup Equivalence of a Measuring Instrument: A Walk through the Process. Psicothema, 20, 872-882.

[19] Browne, M.W. and Cudeck, R. (1993) Alternative Ways of Assessing Model Fit. In: Bolle, K.A. and Long, J.S., Eds., Testing Structural Models, Sage, Newbury Park, 136-162.

[20] Hair, J., Black, W., Babin, B., Anderson, R. and Tatham, R. (2006) Multivariate Data Analysis. 6th Edition, Pearson Prentice Hall, Upper Saddle River.

[21] Bentler, P.M. (2005) EQS 6 Structural Equations Program Manual. Multivariate Software, Encino.

[22] Cheung, G.W. and Rensvold, R.B. (2002) Evaluating Goodness-of-Fit Indexes for Testing Measurement Invariance. Structural Equation Modeling, 9, 233-255. http://dx.doi.org/10.1207/S15328007SEM0902_5

[23] Little, T.M. (1997) Mean and Covariance Structures (MACS) Analyses of Cross-Cultural Data: Practical and Theoretical Issues. Multivariate Behavioral Research, 32, 53-76. http://dx.doi.org/10.1207/s15327906mbr3201_3 


\section{Appendix A: Factors and Items of the Short Form YES-S}

\section{Personal and Social Skills:}

I became better at sharing responsibility

I learned that working together requires some compromising

I learned to be patient with other group members

I learned how my emotions and attitude affect others in the group

\section{Cognitive Skills:}

I have improved: Skills for finding information

I have improved: Academic skills (reading, writing, math, etc.)

I have improved: Computer/internet skills

I have improved: Artistic/creative skills

\section{Goal Setting:}

I learned to find ways to achieve my goals

I set goals for myself in this activity

I learned to consider possible obstacles when making plans

Observed how others solved problems and learned from them

\section{Initiative:}

I learned to push myself

I learned to focus my attention

I put all my energy into this activity

I have improved athletic/physical skills

\section{Negative Experiences:}

Adult leaders in this activity are controlling and manipulative

Adult leaders intimidate me

Adult leaders make personal comments that I find upsetting

Adult leaders encouraged me to do something I believed morally wrong

Youth in this activity got me into drinking alcohol or using drugs

I was treated differently because of my gender, race, ethnicity, disability, or sexual orientation 